\title{
Energy balance and occupational activity as predictors of obesity risk in women.
}

\section{Abstract}

Reduced occupational energy expenditure and increased energy intake are important contributors to the increasing prevalence of obesity. The aim of this study was to examine whether sedentary occupations, and specific indicators of energy intake and expenditure are associated with obesity risk in Australian women. Data were from 3,444 participants in the Australian Longitudinal Study on Women's Health, who reported their weight, dietary intake, physical activity and occupation in 2009 (at age 31-36), and weight in 2012. Participants were categorised as having a 'less sedentary' or 'sedentary work', based on occupation and activity patterns at work. Multivariate models were conducted to examine the odds of being obese ( $>30$ body-mass index) and risk of obesity in the two occupational groups based on energy balance factors (diet and physical activity). Models were adjusted for major confounders (smoking, education, income, number of children).There was no significant difference in the prevalence of obesity between groups $(20.3 \%$ less sedentary vs $22.7 \%$ sedentary work, $p=0.11)$ at baseline. Being in the highest total energy intake tertile, saturated fat intake $>35 \mathrm{~g} / \mathrm{d}$ and drinking 3 or more sugar-sweetened beverages per week increased the odds of being obese in both groups. But to a higher extent in 'less sedentary work' (OR 2.11 95\%CI 1.41-3.19; OR 3.04 95\%CI 2.09-4.45; 2.07 95\%CI 1.45-2.97, respectively). High physical activity (> 1000MET.min/week) was consistently associated with lower odds of being obese (OR $0.6495 \%$ CI $0.43-0.97$ 'less sedentary'; OR 0.58 95\% CI 0.36-0.93 'sedentary work') but lower incidence of obesity only in 'less sedentary work' group (IRR $0.5295 \%$ CI $0.30-0.88$, absolute risk 14\%). High sugar-sweetened beverages increased the incidence of obesity only in this group (IRR $1.7295 \%$ CI 1.08-2.73, absolute risk 23\%). Having a sedentary work per se did not play a major role in obesity prevalence and risk in women. Instead, high saturated fat and SSB intake, and physical inactivity remained the major contributors to obesity prevalence and risk, particularly for those in less sedentary jobs.

\section{Conflict of Interest}

There is no conflict of interest 